\title{
Reduced Operating Time but Not Blood Loss With Cruciate Retaining Total Knee Arthroplasty
}

\author{
Dinu Vermesan ${ }^{\mathrm{a}, \mathrm{i}}$, Ilie Trocan ${ }^{\mathrm{a}}$, Radu Prejbeanu ${ }^{\mathrm{a}}$, Dan V Poenaru ${ }^{\mathrm{a}}$, Horia Haragus ${ }^{\mathrm{a}}$, Damian Gratian ${ }^{\mathrm{b}}$, \\ Massimo Marrelli ${ }^{c, d}$, Francesco Inchingolo ${ }^{\mathrm{e}}$, Monica Caprio ${ }^{\mathrm{f}}$, Raffaele Cagiano ${ }^{\mathrm{f}, \mathrm{h}}$, \\ Marco Tatulloc, g, h
}

\begin{abstract}
Background: There is no consensus regarding the use of retaining or replacing cruciate implants for patients with limited deformity who undergo a total knee replacement. Scope of this paper is to evaluate whether a cruciate sparing total knee replacement could have a reduced operating time compared to a posterior stabilized implant.
\end{abstract}

Methods: For this purpose, we performed a randomized study on 50 subjects. All procedures were performed by a single surgeon in the same conditions to minimize bias and only knees with a less than 20 varus deviation and/or maximum $15^{\circ}$ fixed flexion contracture were included.

Results: Surgery time was significantly shorter with the cruciate retaining implant $(\mathrm{P}=0.0037)$. The mean duration for the Vanguard implant was 68.9 (14.7) and for the NexGen II Legacy was 80.2 (11.3). A higher range of motion, but no significant Knee Society Scores at 6 months follow-up, was used as controls.

Conclusions: In conclusion, both implants had the potential to assure great outcomes. However, if a decision has to be made, choosing a cruciate retaining procedure could significantly reduce the surgical time. When performed under tourniquet, this gain does not lead to reduced blood loss.

Keywords: Incomplete tears; Meniscus in situ; Anatomic single bun-

Manuscript accepted for publication December 11, 2014

aDepartment of Orthopedics and Trauma, University of Medicine and Pharmacy "Victor Babes", Timisoara, Romania

bVest University "Vasile Goldis", Arad, Romania

${ }^{\circ}$ Calabrodental Clinic, Maxillofacial Unit, Crotone, Italy

${ }^{\mathrm{d}}$ Marrelli Hospital, Orthopedics and Traumatology Unit, Crotone, Italy

eDepartment of Interdisciplinary Medicine, Medical Faculty, University of Bari "Aldo Moro", Italy

fDepartment of Biomedical Sciences and Human Oncology, Medical Faculty, University of Bari "Aldo Moro", Italy

"Tecnologica Research Institute, Biomedical Section, Crotone, Italy

${ }^{\mathrm{h}}$ These authors contributed equally to this research paper.

${ }^{i}$ Corresponding Author: Dinu Vermesan, University of Medicine and Pharmacy "Victor Babes", Timisoara, Romania.

Email: d.vermesan@libero.it; dinu@vermesan.ro

doi: http://dx.doi.org/10.14740/jocmr2048w dle; Anterior cruciate ligament reconstruction

\section{Introduction}

Total knee replacement is one of the most successful currently performed arthroplasty. National registries predict a constant rise in the number of their annual procedures. There is still a constant debate regarding the use of cruciate retaining or replacing implants in patients with limited deformity. While there are subtle different benefits for each type, the overall result has always been proved to be the same. This has been previously studied by comparing the cruciate retaining and replacing implant of the same system with regard to functional outcome AMK [1], NexGen [2], Genesis II [3] and range of motion (ROM) $[4,5]$.

All conclude that, at a minimum of 2 years follow-up, functional scores (Knee Society Score (KSS), Western Ontario and McMaster Universities Arthritis Index (WOMAC), and SF-36) were similar but with a clinical not significant increase in $\mathrm{ROM}$ with the posterior replacing design. These findings are maintained even when comparisons were made in bilateral knees [6], mobile bearing [7] and high flexion implant designs [8]. The most common presentation, for knee arthroplasty, is a patient with limited varus deformity, generated by the higher prevalence of arthritis in the medial compartment. The relatively limited coronal plane deviation at surgery is determined by the accompanying intense clinical symptoms and high availability of the procedure. Another perspective is the high strain on the current health care systems worldwide which emphasizes better management and efficient use of resources. The posterior cruciate ligament replacing implants offer a more predictable procedure and outcomes, in comparison to their preservation. Nevertheless, this comes with a more invasive resection on the femur and, sometimes, with a more laborious procedure. We therefore aimed to investigate whether, or not, a cruciate retaining total knee replacement could be a more efficient approach for both provider and patient in most cases. The primary objective of our study was to evaluate if there is a significant difference in operating time between the two procedures with equivalent outcomes. The secondary objective was to determine if this also leads to a reduced perioperative blood 
Table 1. Descriptive Statistics of the Patient's Data at the Point of Index Surgery

\begin{tabular}{llll}
$\mathbf{N}$ & $\mathbf{M} / \mathbf{F}$ & Age (SD) & BMI (SD) \\
\hline $\mathrm{CR}=25$ & $15 / 10$ & $68.8(6.9)$ & $32.6(7.1)$ \\
$\mathrm{PS}=25$ & $22 / 3$ & $68.4(6.3)$ & $33.4(7.5)$ \\
\hline
\end{tabular}

$\mathrm{M} / \mathrm{F}$ : male and female number; SD: standard deviation of the mean for 95\% confidence interval; BMI: body mass index.

loss with the cruciate retaining implant.

\section{Material and Methods}

For this purpose, we performed a randomized study on 50 subjects consecutively enrolled over 16 months in a University Hospital. All surgical procedures were performed by a single experienced knee surgeon in the same operating room and assisted by the same team in order to minimize variables. Both males and females were considered eligible if they were older than 55 years, could comply with the postoperative follow-up visits and had a varus deviation of less than 20 and/or fixed flexion contracture of maximum $15^{\circ}$. Involved subjects have given their informed consent and this study protocol has been approved by the University of Medicine and Pharmacy "Victor Babes" Timisoara Committee on human research.

Preoperative planning was done using standard AP and lateral non-weight bearing radiographs. When in doubt, either skyline axial view of the patella, AP weight-bearing or whole leg CT scanograms were added as needed. Both primary and inflammatory diseases were included. Patients with previous contralateral knee replacement were also deemed eligible. A clinical significant lower limb deformity, posttraumatic or valgus knee arthritis or any general condition (neurologic, and inflammatory) which interfered with the patient's potential ability to ambulate unassisted, were conditions for exclusion. Patient demographics are summarized in Table 1.

All procedures used either cruciate sparing Vanguard ${ }^{\circledR} \mathrm{CR}$ (Biomet) or cruciate replacing NexGen ${ }^{\circledR}$ Legacy II PS (Zimmer) implants. The femoral osteotomies were performed using intramedullary guidance and posterior (condyle) referencing. The box cuts for the posterior stabilized implant were done without a reciprocal saw. A default setting of $6^{\circ}$ of valgus and $3^{\circ}$ of external rotation for the femoral component were used for both implants. The tibial cut used extramedullary guides. For the cruciate retaining implants, a posterior slope resembling local anatomy, allowed a proper gap balancing determined by using a preoperative lateral X-ray and an intraoperative inclination of the lateral plateau [9]. For the posterior stabilized implant, we aimed for a neutral slope to the tibial axis. The implants were cemented in a single stage. The patella was never resurfaced. Five instrument trays were used with the cruciate sparing implant in comparison to seven for the cruciate replacing. The same medial para-patellar approach was used for all cases with eversion of the patella. All surgeries were performed with transient ischemia by use of standard $400 \mathrm{~mm} \mathrm{Hg}$ band pressure. The band was inflated before skin incision after a minimum of 3 min of postural drainage. It was deflated after wound closure using three layers of non-absorbable interrupted sutures and compressive (elastic bandage) dressing. The drain was clamped for the first $2 \mathrm{~h}$. All patients underwent the same postoperative physical therapy regimen started on the second day. Thromboprophylaxis was started on the evening of the surgical session and continued for 28 days using a single daily injection of low molecular heparin [10]. Perioperative bleeding was measured as hemoglobin dropping on the surgical day (morning to evening). Based on a retrospective analysis of the tourniquet times for both procedures, it was estimated $15 \mathrm{~min}$ standard deviation. We therefore computed a sample size of 25 knees for each group ( $80 \%$ power) and a significance level less than 0.05 (two-tailed $t$-test). A predetermined randomization list allowed us to perform similar preoperative planning for all cases. Continuous data were analyzed using descriptive statistics and unpaired $t$-test to compare the two means. Data processing was performed using GraphPad Prism 6 (GraphPad Software, Inc.). The study was approved by our hospital ethics committee and all measures were taken to ensure patients for safety and privacy, including intervention if one of the techniques proved unequivocal superiority. Clinical evaluation was made using the KSS [11].

Together with the WOMAC and ROM, these are the most
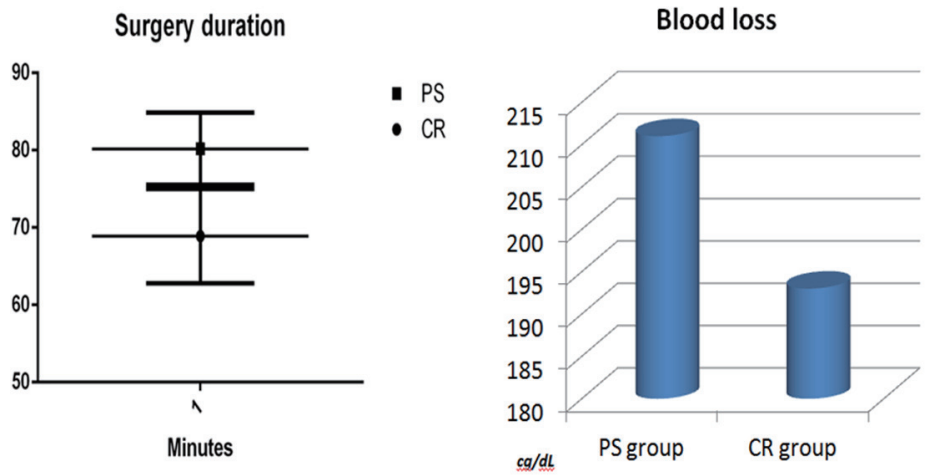

Figure 1. Chart distribution of operating time comparison. 
Table 2. Data Collected at 6 Months Follow-Up

\begin{tabular}{lll}
$\mathbf{N}$ & KSS (SD) & ROM (SD) \\
\hline $\mathrm{CR}=25$ & $83.4(8.5)$ & $100(10)$ \\
$\mathrm{PS}=25$ & $86.1(5.7)$ & $110(15)$ \\
$\mathrm{P}$ & 0.1934 & 0.0079 \\
\hline
\end{tabular}

KSS: Knee Society Score average of the means for symptoms and function; ROM: range of motion; SD: standard deviation of the mean for $95 \%$ confidence interval.

widely used outcomes to compare cruciate sparing and replacing implants [12]. A single 6 months follow-up visit was used to determine similarity of final outcome for both implants, since these was not an objective of our study, but only a control measure; the 6 months results have shown to be predictive of the final (2 years) outcome $[4,5]$. An unofficial local translation of the scores was administered at 1 year postoperatively in order to determine the outcome. All authors agreed on a local translation of the original KSS scale as described by Likertscale patient questionnaires forms (www.orthopaedicscore. com) for a theoretical floor of 0 and ceiling of 100. The patients were instructed to answer all questions and to complete the forms with assistance (when needed) from one of the authors. All ROM measurements were evaluated in supine position as maximal passive flexion quantified with a hand held goniometer.

\section{Results}

The surgery time was significantly shorter with the cruciate retaining implant $(\mathrm{P}<0.005)$. The mean duration for the Vanguard implant was 68.9 (14.7) and for the NexGen II Legacy was 80.2 (11.3). The perioperative drop in hemoglobin was not significantly $(\mathrm{P}=0.4948)$ higher in the PS group: $2.11(0.94)$ compared to $1.93(0.91) \mathrm{g} / \mathrm{dL}$. This was also the case with regard to blood transfusion: four cases in the PS and three in the CR group received one unit of red blood cells (Fig. 1). All patients returned for the 6 months follow-up (5 - 7 months). The collected data are scheduled in Table 2.

Patients from both groups had results which could be considered favorable when compared to expected follow-up after such a procedure. After 6 months, a follow-up was maintained in the same manner as for the rest of the patients. Survivorship and long-term outcome are still being followed on a yearly basis.

One case, in the required re-intervention during this period for wound infection, has been solved with debridement, aspirative drainage and antibiotic therapy.

Three cases, in the CR group, and one in the PS group, evidenced stiff knees at 6 months (ROM less than $80^{\circ}$ ).

\section{Discussion}

In our study group, the cruciate retaining implant had a clear advantage over the posterior stabilized, with respect to operat- ing time. Our data come from a single surgeon cohort, using the same technique for all cases, as they offered a consistent uniformity of comparisons together with minimized bias. The main author had consistent experience with both implants. To our knowledge, this is the only report focusing on the duration of surgery and possible benefit to blood loss between CR and PS total knee replacements. The most important purpose of our study is to compare the two used systems which consequently involve also the instrumentation and surgical technique used. Looking forward, it is also possible to improve surgical time and lead to a more efficient management with comparative results between different total knee systems.

When we look at literature reviews, there is a unanimously finding of better postoperative ROM in favor of posterior cruciate replacement. However, this is generally reported as around $5^{\circ}$ and no differences in the KSS, WOMAC, component alignment, tibial posterior slope, joint line and incidence of complications [13]. This leads us to conclude that the reported gain in amplitude of movement has no effects on the clinical outcome, knee function or patient satisfaction, which are the most important parameters for any arthroplasty procedure. Similar outcomes, with reduced ROM, have led to studies evaluating comparative biomechanics of the two procedures. There are differences that probably contribute together for final results. Retention of the posterior cruciate ligament does not appear to significantly improve proprioception and balance [14]. In addition, Victor et al found that femoral roll-back was greater and more consistent with cruciate-substituting and this was predictive of the maximum flexion [15]. In contrast, a different study observed that posterior cruciate-substituting only showed a significant increase in posterior femoral translation after $90^{\circ}$ [16]. Unconstrained implants are associated with less joint line changes [17], are superior in achieving equalized rectangular extension and flexion gaps [18] and can even produce improved survival [19]. By comparison, posterior stabilized knees are less susceptible to subtle changes such as the magnitude of posterior condylar offset which can correlate with a postoperative change in flexion in cruciate retaining systems [20,21]. Bell et al found that intraoperative tourniquet release was associated with a significantly lower reduction in hemoglobin with respect to its release after wound closure [22]. In addition, a recent meta-analysis found that tourniquet release after wound closure could shorten the operation time but may not reduce the blood loss [23]. Our hypothesis was that, in similar conditions, a reduced surgical time might also reduce perioperative blood loss. This was not found to be significant, mainly because the operative gain was under transient ischemia and postoperative hemorrhage weight more towards total loss. In addition, the drop in hemoglobin was not adjusted for dilution.

\section{Conclusions}

We conclude that both implants have the potential to assure great outcomes. However, if a decision has to me made, choosing a cruciate retaining procedure can significantly reduce the surgical time. When performed under tourniquet, this gain 
does not lead to reduced blood loss.

\section{Acknowledgement}

Thanks are given to Cristina Nuta, MD for contributing with the postoperative physical therapy for the cases.

\section{Competing Interests}

The authors declare that they have no competing interests.

\section{Author Contributions}

VD and TM have made substantial contributions to conception and design of this paper. CR, CM, TI and MM have contributed to draft the manuscript. GD and AA have collected, revised and managed the data inside the text. PDL and PR have revised the literature sources and have followed and documented the follow-up. VD , HH, PR and GD participated to surgical activities. All the authors have approved this manuscript.

\section{References}

1. Clark CR, Rorabeck CH, MacDonald S, MacDonald D, Swafford J, Cleland D. Posterior-stabilized and cruciateretaining total knee replacement: a randomized study. Clin Orthop Relat Res. 2001;(392):208-212.

2. Tanzer M, Smith K, Burnett S. Posterior-stabilized versus cruciate-retaining total knee arthroplasty: balancing the gap. J Arthroplasty. 2002;17(7):813-819.

3. Harato K, Bourne RB, Victor J, Snyder M, Hart J, Ries MD. Midterm comparison of posterior cruciate-retaining versus -substituting total knee arthroplasty using the Genesis II prosthesis. A multicenter prospective randomized clinical trial. Knee. 2008;15(3):217-221.

4. Chaudhary R, Beaupre LA, Johnston DW. Knee range of motion during the first two years after use of posterior cruciate-stabilizing or posterior cruciate-retaining total knee prostheses. A randomized clinical trial. J Bone Joint Surg Am. 2008;90(12):2579-2586.

5. Ishii Y, Noguchi H, Takeda M, Sato J, Toyabe S. Prediction of range of motion 2 years after mobile-bearing total knee arthroplasty: PCL-retaining versus PCL-sacrificing. Knee Surg Sports Traumatol Arthrosc. 2011;19(12):20022008.

6. Maruyama S, Yoshiya S, Matsui N, Kuroda R, Kurosaka M. Functional comparison of posterior cruciate-retaining versus posterior stabilized total knee arthroplasty. J Arthroplasty. 2004;19(3):349-353.

7. Aglietti P, Baldini A, Buzzi R, Lup D, De Luca L. Comparison of mobile-bearing and fixed-bearing total knee arthroplasty: a prospective randomized study. J Arthroplasty. 2005;20(2):145-153.

8. Kim YH, Choi Y, Kwon OR, Kim JS. Functional outcome and range of motion of high-flexion posterior cruciateretaining and high-flexion posterior cruciate-substituting total knee prostheses. A prospective, randomized study. J Bone Joint Surg Am. 2009;91(4):753-760.

9. Bae DK, Song SJ, Yoon KH, Noh JH, Moon SC. Comparative study of tibial posterior slope angle following cruciate-retaining total knee arthroplasty using one of three implants. Int Orthop. 2012;36(4):755-760.

10. Prejbeanu R, Vermesan H, Dragulescu SI, Vermesan D, Motoc A, Sabatini R, Santacroce L, et al. Thromboembolic risk after knee endoprosthesis. Eur Rev Med Pharmacol Sci. 2007;11(5):297-300.

11. Insall JN, Dorr LD, Scott RD, Scott WN. Rationale of the Knee Society clinical rating system. Clin Orthop Relat Res. 1989;(248):13-14.

12. Li N, Tan Y, Deng Y, Chen L. Posterior cruciate-retaining versus posterior stabilized total knee arthroplasty: a meta-analysis of randomized controlled trials. Knee Surg Sports Traumatol Arthrosc. 2014;22(3):556-564.

13. Luo SX, Zhao JM, Su W, Li XF, Dong GF. Posterior cruciate substituting versus posterior cruciate retaining total knee arthroplasty prostheses: a meta-analysis. Knee. 2012;19(4):246-252.

14. Swanik CB, Lephart SM, Rubash HE. Proprioception, kinesthesia, and balance after total knee arthroplasty with cruciate-retaining and posterior stabilized prostheses. J Bone Joint Surg Am. 2004;86-A(2):328-334.

15. Victor J, Banks S, Bellemans J. Kinematics of posterior cruciate ligament-retaining and -substituting total knee arthroplasty: a prospective randomised outcome study. J Bone Joint Surg Br. 2005;87(5):646-655.

16. Most E, Zayontz S, Li G, Otterberg E, Sabbag K, Rubash HE. Femoral rollback after cruciate-retaining and stabilizing total knee arthroplasty. Clin Orthop Relat Res. 2003;(410):101-113.

17. Pang HN, Yeo SJ, Chong HC, Chin PL, Chia SL, Lo NN. Joint line changes and outcomes in constrained versus unconstrained total knee arthroplasty for the type II valgus knee. Knee Surg Sports Traumatol Arthrosc. 2013;21(10):2363-2369.

18. Matsumoto T, Kubo S, Muratsu H, Matsushita T, Ishida K, Kawakami Y, Oka S, et al. Different pattern in gap balancing between the cruciate-retaining and posteriorstabilized total knee arthroplasty. Knee Surg Sports Traumatol Arthrosc. 2013;21(10):2338-2345.

19. Abdel MP, Morrey ME, Jensen MR, Morrey BF. Increased long-term survival of posterior cruciate-retaining versus posterior cruciate-stabilizing total knee replacements. J Bone Joint Surg Am. 2011;93(22):2072-2078.

20. Arabori M, Matsui N, Kuroda R, Mizuno K, Doita M, Kurosaka M, Yoshiya S. Posterior condylar offset and flexion in posterior cruciate-retaining and posterior stabilized TKA. J Orthop Sci. 2008;13(1):46-50.

21. Vermesan D, Prejbeanu R, Laitin S, Georgianu V, Haragus $\mathrm{H}$, Nitescu S, Tatullo M, et al. Meniscal tears left in situ during anatomic single bundle anterior cruciate ligament reconstruction. Eur Rev Med Pharmacol Sci. 2014; 18(2):252-256.

22. Bell TH, Berta D, Ralley F, Macdonald SJ, McCalden 
RW, Bourne RB, Rorabeck CH, et al. Factors affecting perioperative blood loss and transfusion rates in primary total joint arthroplasty: a prospective analysis of 1642 patients. Can J Surg. 2009;52(4):295-301.
23. Tai TW, Lin CJ, Jou IM, Chang CW, Lai KA, Yang CY. Tourniquet use in total knee arthroplasty: a meta-analysis. Knee Surg Sports Traumatol Arthrosc. 2011;19(7):11211130. 